\title{
Acute cholecystitis developed immediately after thoracic kyphoplasty -A case report-
}

\author{
Sang-Bum An, Jiyeon Yim, Eunjung Kim, Jae-Hyuck Shin, Soo Young Park, and Sang Chul Lee \\ Department of Anesthesiology and Pain Medicine, Seoul National University Hospital, Seoul, Korea
}

Postoperative acute cholecystitis is a rare complication of orthopaedic surgery and is unrelated to the biliary tract. In particular, in the case of immediate postoperative state after surgery such as kyphoplasty at the thoracic vertebra, symptoms related to inflammation mimic those of abdominal origin, so the diagnosis and the treatment of acute cholecystitis can be delayed leading to a fatal outcome. It is important that physicians should be aware of the postoperative patient's condition in order to make an early diagnosis and determine treatment. (Korean J Anesthesiol 2012; 63: 266-269)

Key Words: Acute cholecystitis, Complication, Kyphoplasty, Postoperative.

Postoperative acute cholecystitis is a well-known complications of general surgery that includes abdominal surgery [1-3]. It is related to the operation field near and around the biliary tract primarily, but in a surgery that does not involve the biliary tract, such as an orthopedic surgery, it only occurs rarely $[2,3]$. When once postoperative acute cholecystitis occurs, progression of the disease is fast, obscure, and fulminating. Rapid progression to sepsis and perforation of the gall bladder may eventually lead to death $[2,3]$.

On the other hand, balloon kyphoplasty, including vertebroplasty, is used for management compression fracture of vertebra and concordant pain as minimally invasive percutaneous techniques [4-8]. Even though they have low incidence of complications, several related complications have been reported, such as infection [7-10].

In view of postoperative infection, there are the usual red flag signs of inflammation, such as redness, fever, or tenderness at the site $[7,9,10]$. However, it may be difficult to differentiate from other origin immediately after the procedure. Since, in particular, pain from the thoracic vertebra can be presented as abdominal pain [3], physicians should be aware of patients' general condition after the procedure.

Herein, we report a case of postoperative acute cholecystitis following balloon kyphoplasty at the thoracic vertebra.

\section{Case Report}

A 70-year-old female visited to our pain center with severe

Received: June 14, 2011. Revised: 1st, November 2, 2011; 2nd, December 6, 2011. Accepted: December 7, 2011.

Corresponding author: Soo Young Park, M.D., Department of Anesthesiology and Pain Medicine, Seoul National University Hospital, 110, Daehang-ro, Jongno-gu, Seoul 110-744, Korea. Tel: 82-2-2072-0881, Fax: 82-2-763-9390, E-mail: soo02@snu.ac.kr

(c) This is an open-access article distributed under the terms of the Creative Commons Attribution Non-Commercial License (http:// creativecommons.org/licenses/by-nc/3.0/), which permits unrestricted non-commercial use, distribution, and reproduction in any medium, provided the original work is properly cited. 
lower back pain. The pain intensity was 8 out of $10(0=$ no pain, 10 = worst imaginable pain) measured by the Visual Analog Scale (VAS). Even though her symptoms developed after a slip-down injury one month before, the pain was intractable to conservative treatment and increased with any movement. $\mathrm{X}$-ray imaging and MRI scan revealed a newly developed compression fracture at the T12 vertebral body with loss of height leading to kyphosis, otherwise within normal range on work-up even though the patient had hypertension, diabetes mellitus, and a gallstone on past medical history.

The patient was offered a balloon kyphoplasty under conscious sedation. After obtaining informed consent from the patient and her family, the balloon kyphoplasty procedure was performed under local anesthesia. If necessary, fentanyl $(25-50 \mu \mathrm{g})$ was injected intermittently for pain relief; no other analgesics or sedatives were administered. The procedure ended without any incident and the pain intensity was decreased from the initial 8 to 3 out of 10 on VAS immediately after the kyphoplasty. For observation, the patient was admitted to the hospital with the 1-day course as our routine post-procedural management protocol.

In the morning of the post operative day 1 , the patient did not complain of back pain, but felt a chilling sensation and intermittent sweating with a fever up to $39^{\circ} \mathrm{C}$. On the physical examination, there was neither tenderness nor heating sense on the site of procedure, however, the patient complaint of vague whole abdominal discomfort.

Emergency laboratory results were as follows: white blood cell count, $14.4 \times 10^{3} / \mathrm{L}$; erythrocyte sedimentation rate, 102 $\mathrm{mm} / \mathrm{h}$; C-reactive protein, $29.39 \mathrm{~g} / \mathrm{L}$; total bilirubin, $4.4 \mathrm{mg} / \mathrm{dL}$.

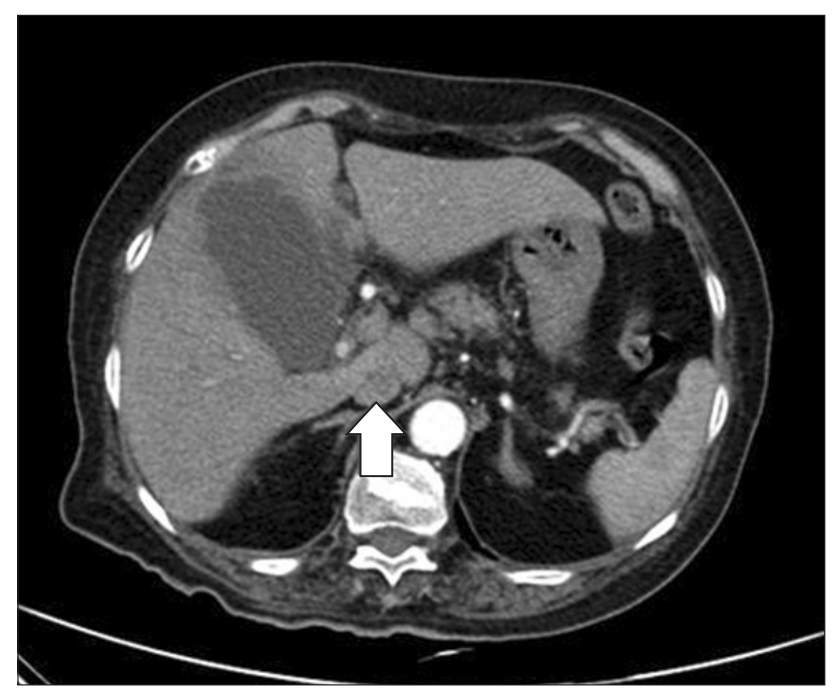

Fig. 1. Precontrast abdominal CT scan shows a gangrenous cholecystitis with confined pericholecystic fluid collection and multiple liver microabscesses (arrow).
Other blood biochemistry tests were normal. On the physical exam at the evening, the patient had a pain in the right upper quadrant revealing positive Murphy's sign. Abdominal CT scan showed acute cholecystitis and multiple liver microabscesses (Fig. 1). Peripheral blood cultures were positive for Klebsiella Pneumoniae, and liver abscess aspirates cultures were positive for Klebsiella Pneumoniae and quinolone resistant Escherichia Coli.

Emergency percutaneous transhepatic gallbladder drainage (PTGBD) was carried out (Fig. 2), and the patient was treated with analgesics and antibiotics. The acute state was gradually subsided over a period of 3 weeks and a laparoscopic cholecystectomy was performed. The patient was discharged without any complication.

\section{Discussion}

This case report showed the importance of the physician's awareness to prevent a severe outcome derived from a complication of an unrelated part of the body in post-operative period.

In general, complications of kyphoplasty, including infection are well known $[7,9,10]$. However, postoperative acute cholecystitis, associated with kyphoplasty, has not been reported to our knowledge. Despite the importance of early recognition for treatment [1-3], postoperative acute cholecystitis may not be easy to diagnose properly in the early perioperative period, especially where the field of operation was unrelated to the biliary track. However, several studies have shown that orthopedic operations were the second most common

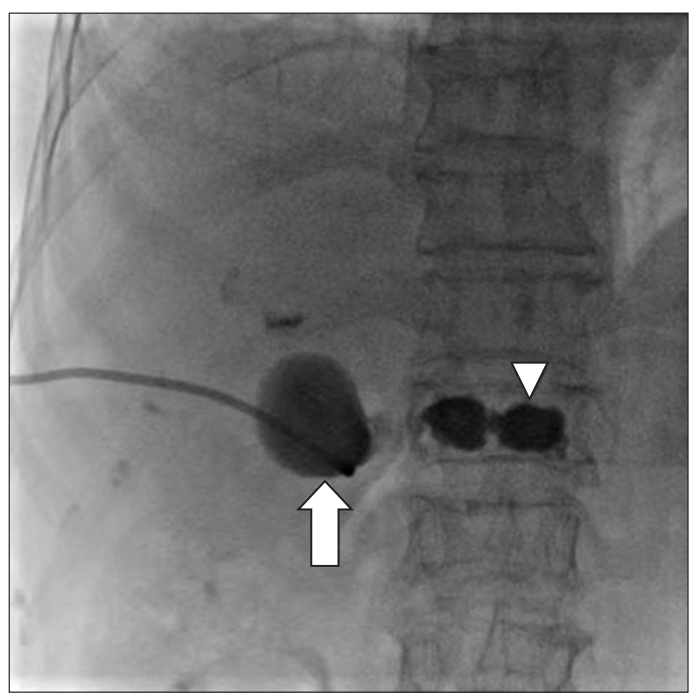

Fig. 2. The simple x-ray shows the kyphoplasty state of a compressed T12 body with the catheter of the percutaneous cholecystostomy (PTGBD, arrow). The gall-bladder and the level of kyphoplasty, T12 (arrowhead), are placed on almost the same transverse plane. 
cause $(16.5 \%)$ of postoperative acute cholecystitis next to the gastrointestinal and abdominal operations (40\%) [1-3].

There is general agreement that the usual form of acute cholecystitis is nearly always due to obstruction of the cystic duct by an impacted stone. Even though the pathogenic factors of the postoperative acute cholecystitis are uncertain [4], bile stasis has been demonstrated as a factor in an animal study. Several factors have been suggested as contributing to bile stasis such as fasting, anesthesia, dehydration, fever, and narcotics use for pain relief $[1,4]$.

Compared with ordinary acute cholecystitis, postoperative acute cholecystitis can occur in old age with vague symptoms, but there is a higher mortality rate [2]. So, the key to successful treatment is early diagnosis, simultaneous intensive preparation with fluids and antibiotics, and immediate drainage or cholecystectomy, as soon as possible [3].

In this case, the patients complained of vague abdominal pain with chilling sensation, first. Since the patient had a kyphoplasty at the T12 level, differential diagnosis of her symptoms was mainly focused on complications related to the procedure. However, there were no sign of skin infection at the site of procedure, no referred pain, or neurologic deficit along the dermatome of T12 somatosensory level.

Fortunately, part of the patient's medical history included an asymptomatic gallstone and Murphy's sign supported the diagnosis of acute cholecystitis on physical examination. Visceral pain from the gallbladder in biliary colic or cholecystitis felt in the lower chest and upper abdominal wall due to nerve supply to dermatomes (T5 to T9) [11]. If the level of kyphoplasty due to compression fracture was at the mid-thoracic vertebra, such as the $\mathrm{T} 6$ or $\mathrm{T} 7$, the diagnosis might be difficult and delayed because of somatosensory innervations or referred pain.

Because gallbladder and the level of kyphoplasty, T12, were almost placed on the same transverse plane in the radiographic image of gallbladder after performing PTGBD as shown in Fig. 2 , we considered the possibility of infection as a postoperative complication of kyphoplasty. From a blood culture and a liver abscess culture, Klebsiella Pneumoniae and quinolone resistant Escherichia Coli were isolated. Although various pathogens could be isolated in case of postoperative infection [10], we excluded the direct infection from the field of procedure since the most common pathogen via needle pathway was Staphylococcus aureus [12].

In our case, even though the patients had a history of gallbladder stones, she was elderly and her initial symptom was vague, with a fever of $39^{\circ} \mathrm{C}$. Besides, there was no evidence of the inflammatory phase in the blood test at the day of surgery. Since the patient had a kyphoplasty, diagnosis of her symptoms was mainly focused on the complications related to the procedure. However, there were no sign of skin infection at the site of procedure and no referred pain or neurologic deficit along the dermatome of T12 level. Also, back pain was relieved after the procedure with pain measurement on VAS ranging from 8 to 3. Several hours later, the patient's pain was localized to the right upper quadrant and her Murphy's sign was positive. Because fasting time had been prolonged before procedure, it was likely that her dehydration had worsened. Hence, under the circumstances of older age, prolonged fasting state leading to dehydration, underlying gallbladder stone problems, and postoperative status, the patient might had a higher change of getting cholecystitis.

In the western culture, 10 to 20 percent of the population has gallstones. Furthermore, the rate of having gallstones is increasing to $30 \%$ for the population in their 60 s and to $60 \%$ of the population in their 80 s [13]. Meanwhile, as the number of geriatric patients increases, the number of surgery of geriatric patients is also increasing. Since the elderly are prone to dehydration, prolonged fasting time prior to surgery can worsen the symptom of gallbladder stone. As the western pattern dietary habits, which is characterized by an high intake of red meat, sugary desserts, high-fat foods, and refined grains, are increasing in Korean culture, the number of patients experiencing gallbladder stones is also rising. According to the Korean Health Insurance Review \& Assessment Service, the number of patient with gallbladder stone increased by $29.4 \%$ from 78,000 in 2005 [14] to 101,000 in 2009 [15]. Among the patient with gallbladder stone, $40 \%$ has no symptoms. Considering all these matters, the chance of getting postoperative acute cholecystitis is increased in elderly patients.

Therefore, physicians should be aware that acute cholecystitis must be considered in the differential diagnosis of postoperative complications after procedures even if operative field was not related to biliary track, such as kyphoplasty.

\section{References}

1. Thompson JW 3rd, Ferris DO, Baggenstoss AH. Acute cholecystitis complicating operation for other diseases. Ann Surg 1962; 155: 48994.

2. Ottinger LW. Acute cholecystitis as a postoperative complication. Ann Surg 1976; 184: 162-5.

3. Abrahamson J, Eldar S. Acute cholecystitis after orthopedic operations. Int Orthop 1988; 12: 93-5.

4. Inoue T, Mishima Y. Postoperative acute cholecystitis: a collective review of 494 cases in japan. Jpn J Surg 1988; 18: 35-42.

5. Kang JM, Kim YC, Lee SC. Percutaneous balloon kyphoplasty in vertebral body compression fracture: A case report. Korean J Anesthesiol 2003; 44: 283-6.

6. Joh JY, Bae YG, Kim YH, Lee EH, Lee CJ, Lee SC, et al. Retrograde study on thoracic percutaneous vertebroplasty and kyphoplasty done by transpedicular approach. Korean J Pain 2005; 18: 204-7. 
7. Robinson Y, Tschöke SK, Stahel PF, Kayser R, Heyde CE. Complications and safety aspects of kyphoplasty for osteoporotic vertebral fractures: A prospective follow-up study in 102 consecutive patients. Patient Saf Surg 2008; 2:2. Available from http://creativecommons. org/licenses/by/2.0.

8. Wardlaw D, Cummings SR, Van Meirhaeghe J, Bastian L, Tillman JB, Ranstam J, et al. Efficacy and safety of balloon kyphoplasty compared with non-surgical care for vertebral compression fracture: a randomised controlled trial. Lancet 2009; 373: 1016-24.

9. Jo DH, Park SH, Kim MH, Seol JH. Infectious spondylitis following kyphoplasty: A case report. Korean J Pain 2007; 20: 219-23.

10. Schofer MD, Lakemeier S, Peterlein CD, Heyse TJ, Quante M. Primary pyogenic spondylitis following kyphoplasty: A case report. J Med Case Reports 2011; 5: 101. Available from http://www. jmedicalcasereports.com/content/5/1/101.

11. Dey A, Malik VK. Understanding pain abdomen: Anatomical considerations. The Ganga Ram Journal 2011; 1: 123-7.

12. Reihsaus E, Waldbaur H, Seeling W. Spinal epidural abscess: a meta-analysis of 915 patients. Neurosurg Rev 2000; 23: 175-204.

13. Sakorafas GH, Milingos D, Peros G. Asymptomatic cholelithiasis: is cholecystectomy really needed? A critical reappraisal 15 years after the introduction of laparoscopic cholecystectomy. Dig Dis Sci 2007; 52: 1313-25.

14. Kim CY. 2005 National Health Insurance Statistical Yearbook. Korea, National Health Insurance Corporation. 2006, p 461.

15. Chung HG. 2009 National Health Insurance Statistical Yearbook. Korea, National Health Insurance Corporation. 2010, p 549. 